\title{
Spatial Audio Quality Perception (Part 1): Impact of Commonly Encountered Processes
}

\author{
ROBERT CONETTA ${ }^{1,2}$, TIM BROOKES, ${ }^{1}$ AES Member, FRANCIS RUMSEY, ${ }^{1,3}$ AES Fellow, \\ (robertc@sandybrown.com) (t.brookes@surrey.ac.uk) (fjr@aes.org) \\ SŁAWOMIR ZIELIŃSKI ${ }^{1,4}$, MARTIN DEWHIRST, ${ }^{1}$ AES Associate Member, \\ (slawek.Zieliński@live.co.uk) (martin.dewhirst@surrey.ac.uk) \\ PHILIP JACKSON, ${ }^{1}$ AES Associate Member, SøREN BECH, ${ }^{5}$ AES Fellow, \\ (P.Jackson@surrey.ac.uk) \\ (sbe@bang-olufsen.dk) \\ DAVID MEARES ${ }^{6}$, AND SUNISH GEORGE, ${ }^{1,7}$ AES Associate Member \\ (sunish.george@iis.fraunhofer.de) \\ ${ }^{1}$ University of Surrey, Guildford, UK \\ ${ }^{2}$ now at Sandy Brown Associates LLP, UK \\ ${ }^{3}$ now at Logophon Ltd., Oxfordshire, UK \\ ${ }^{4}$ now at the Technical Schools, Suwatki, Poland \\ ${ }^{5}$ Bang \& Olufsen a/s, 7600 Strüer, Denmark \\ ${ }^{6}$ DJM Consultancy, West Sussex, UK, on behalf of BBC Research, UK \\ ${ }^{7}$ now at Harman Becker Automotive Systems GmbH, Germany
}

\begin{abstract}
Spatial audio processes (SAPs) commonly encountered in consumer audio reproduction systems are known to generate a range of impairments to spatial quality. Two listening tests (involving two listening positions, six 5-channel audio recordings, and 48 SAPs) indicate that the degree of quality degradation is determined largely by the nature of the SAP but that the effect of a particular SAP can depend on program material and on listening position. Combining off-center listening with another SAP can reduce spatial quality significantly compared to auditioning that SAP centrally. These findings, and the associated listening test data, can guide the development of an artificial-listener-based spatial audio quality evaluation system.
\end{abstract}

\section{O INTRODUCTION}

A desire exists to create or reproduce increasingly real and immersive soundfields or listening experiences [1][2][3][4][5]. This can be observed in the functionality of current consumer products (e.g., surround sound "homecinema" systems, DVD video and audio appliances, gaming consoles). Mobile devices such as MP3 players, mobile phones, and tablet computers are becoming increasingly popular and have the potential to deliver binaurally enhanced spatially immersive environments via headphones [6][7]. Furthermore, broadcasters can now deliver spatially enhanced multichannel audio scenes in the form of matrixed 5.1 surround sound via high definition (HD) television broadcasts [8][9].

Multichannel audio codecs are often used to reduce bandwidth requirements but they can have detrimental effects on perceived spatial audio quality [10]; this is particularly apparent under the most band-limited delivery conditions (e.g., online streaming) and where storage space is limited (e.g., mobile phone MP3 players). The delivery format of audio program material is often different from the rendering (reproduction) format: audio is delivered in a format that suits the transmission technology (e.g., HD broadcast, DVD) and can be reformatted for replay over any of a number of reproduction systems (e.g., 2-channel stereo, 5.1); the upmixing and downmixing techniques used for such reformatting can further degrade quality [11][12][13], as can changes made by the consumer to intended loudspeaker positions. Degradations could include changes to source-related attributes such as perceived location, width, distance, and stability and changes to environment-related attributes such as envelopment and spaciousness [6].

The desires, technologies, and consequences outlined above motivate the development of an efficient and effective method for assessing perceived spatial quality, for 
research, for product development, and for quality control. The costs (in terms of time and money) of maintaining a listening panel, and assessing audio quality by formal listening tests, can be prohibitive [14]. A computer model of quality perception could act as an "artificial listener." An artificial-listener-based perceptual evaluation system, while perhaps not completely replacing assessment by human listeners, could however provide an indication of likely perceived audio quality where human assessment would be impractical or impossible.

Current standard algorithms for evaluating perceived sound quality (e.g., PEAQ [15]) focus on impairments to timbral quality such as audio coding distortions, noise, and bandwidth reductions and do not account for the contribution of spatial attributes ${ }^{1}$.

Since the development of PEAQ, Choi et al. [17], George [18], and Seo et al. [19] have created spatially-aware sound quality models but these only consider the degradations resulting from a limited selection of spatial audio processes (SAPs).

The QESTRAL (Quality Evaluation of Spatial Transmission and Reproduction using an Artificial Listener) project aimed to develop an artificial-listener-based evaluation system capable of predicting, for real or virtual multichannel loudspeaker reproduction, the perceived spatial quality degradations resulting from a wider range of SAPs. Metrics and extraction algorithms for a number of spatially-relevant audio features (informed by the body of research in binaural auditory modeling that aims to predict the perception of specific spatial attributes) have already been developed [20][21][22]. The experiments reported in the current paper aim to determine, by way of two listening tests, the degree of perceived overall spatial quality degradation resulting from SAPs commonly encountered in consumer audio reproduction systems and to determine the influences of listening position and source material on that degradation. The intention is (i) to build a quality-annotated database of processed and unprocessed program items; and (ii) to gain qualitative insights into the effects of SAPs on quality. In a follow-up paper these findings and the quality-annotated database will be combined with the previously-developed metrics to build a regression model of perceived spatial audio quality.

\subsection{Spatial Quality}

Spatial audio quality is a global attribute comprising a number of lower level attributes [23]. Past studies by Berg [24], Berg and Rumsey [25], Choisel and Wickelmaier [26], Koivunmiemi and Zacharov [27], Rumsey [6], Rumsey et al. [28], and Zacharov and Koivunmiemi [29, 30] have identified a number of these lower level attributes (e.g., source location, width, depth, envelopment). However, in order to avoid exclusion of potentially-important factors, the current study is not limited to specific previously-identified attributes but, instead, defines spatial quality as the global

\footnotetext{
${ }^{1}$ An adaptation to enable PEAQ to evaluate degradations to spatial quality is under consideration [16].
}

attribute encompassing any and all perceived spatial differences between a reference recording and a processed version.

\section{DESIGN OF LISTENING TESTS}

Two listening tests were conducted to achieve the aims stated above. In each test listeners were required to rate the perceived spatial quality of each of a number of test stimuli, as compared to a reference stimulus. Each test stimulus was a SAP-degraded version of the reference stimulus against which it was compared. For each test stimulus, the average of all its quality ratings was sought for the quality database. The following sections explain the reasons for using two tests and two listening positions, detail the test apparatus, program items, and SAPs and describe the loudness equalization applied and the test method employed.

\subsection{Use of Two Tests and Two Listening Positions}

It is known from previous studies that off-axis listening leads to image skew [31] and that this skew has a negative impact on overall quality [6]. There have been various attempts to widen the acceptable listening area [32][33] but no previous studies have quantified the impact of off-center listening on overall spatial audio quality. The QESTRAL system was intended to be able to evaluate spatial audio quality at both on- and off-center listening positions and so the effects of listening position were investigated. They were considered in two complementary ways, using two listening tests, with the choice of off-center listening position informed by the previous studies cited above and the likely seating positions in a typical domestic listening room.

In listening test 1 , centrally-auditioned SAPs were compared to a centrally-auditioned reference and off-centerauditioned SAPs were compared, separately, to an offcenter-auditioned reference. Thus, alternative listening positions were treated as alternative test conditions under which to evaluate the effects of a wide range of SAPs. This allowed determination of the extent to which the deleterious effects of SAPs might depend on listening position (e.g., one SAP might degrade a centrally-auditioned signal significantly but for an off-center listener the same SAP might leave the reference signal quality relatively unimpaired).

In listening test 2, centrally-auditioned SAPs and offcenter-auditioned SAPs were both compared to a centrallyauditioned reference. Thus, off-center listening was, in effect, treated as an additional SAP combined with the SAP under test. This allowed examination of the resulting compound quality degradation (e.g., moving off-center might significantly degrade the perceived spatial quality of one particular SAP but might make little difference to the quality of another SAP).

\subsection{Listening Test Apparatus}

The listening tests were conducted at the University of Surrey's Institute of Sound Recording (IoSR) in a listening room compliant with ITU-R BS.1116-1 [34] requirements. 


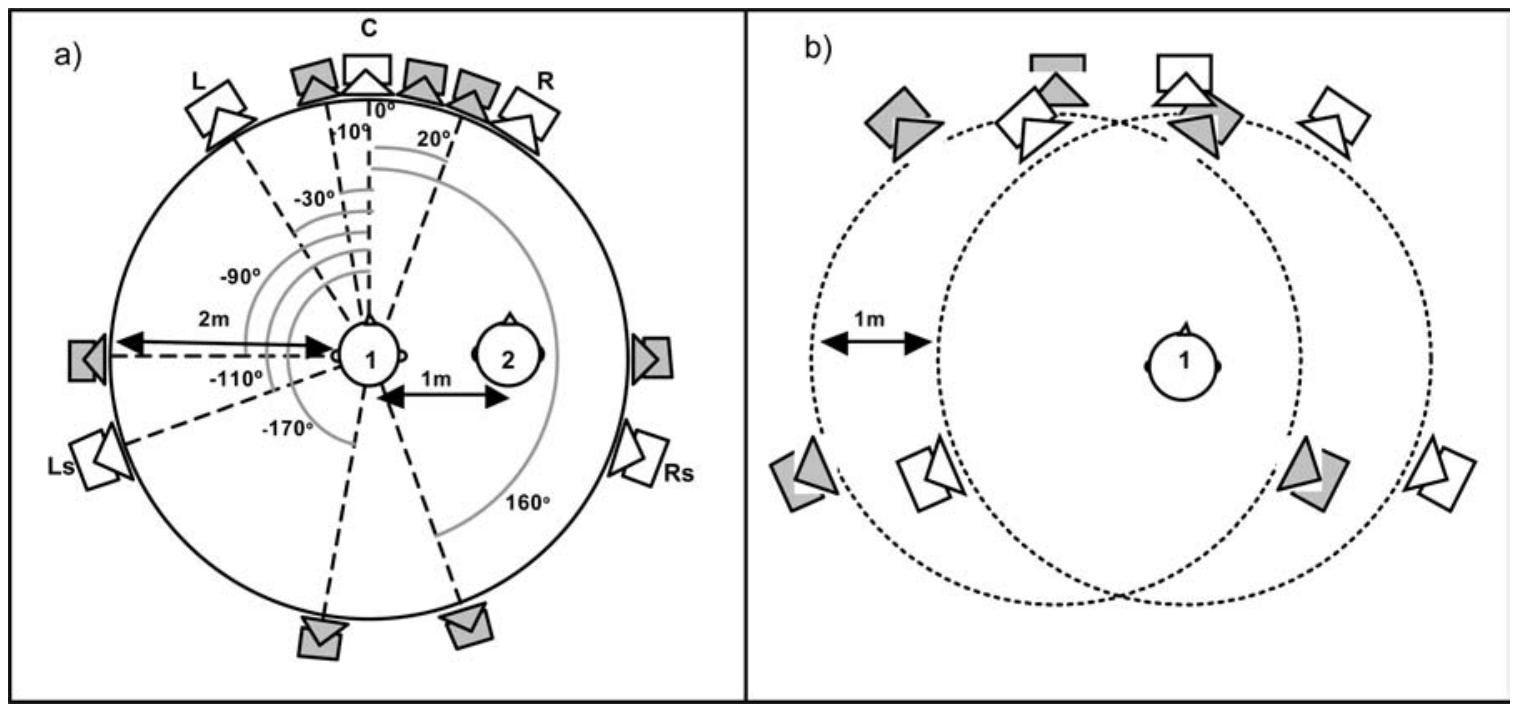

Fig. 1. (a) Listening and loudspeaker positions for listening test 1: core $3 / 2$ array (white) labelled L, C, R, Ls, and Rs. (b) Listening and loudspeaker positions for listening test 2: core 3/2 array (white); off-center array (grey).

Bang and Olufsen Beolab 3 loudspeakers (Frequency response: $50 \mathrm{~Hz}$ to $20 \mathrm{kHz}$ [35]) were used and were concealed from the listener by an acoustically transparent but visually opaque curtain. The high-quality listening room and loudspeakers were chosen in order that the reproduction system should be as transparent as possible, so that the most significant degradations to the program material would be due to the SAPs under test. A room with a poorer acoustic, or lower-quality loudspeakers, would constitute an additional SAP. This could be considered in a future investigation and the effects on quality incorporated into a future version of the QESTRAL system.

For listening test 1 the core playback system comprised five loudspeakers arranged in $3 / 2$ stereo configuration according to the requirements described in ITU-R BS.775-1 [11]; additional loudspeakers were employed for SAPs that required them (Fig. 1). Listening test 2 employed two 5channel loudspeaker systems, one as a reference system with a central listening position (LP1) and one to provide an off-center listening position (LP2) for comparison. Prior to each test all channel gains were calibrated individually to produce the same sound pressure level, at the center of the corresponding loudspeaker system, using a pink noise test signal.

\subsection{Program Material}

SAPs were applied to six 5-channel audio recordings (Table 1). These program items were chosen to span a representative range of ecologically valid audio recordings, likely to be listened to by typical audiences of consumer multichannel audio, while also covering typical genres and spatial audio scene types. For example, the content of program item 1 (TV/sport) is mixed to represent a scene suitable for a television sports broadcast with multichannel audio. There are two commentators panned slightly left and right of the front center position where the television set would likely be. Audience applause and ambience can be heard from $360^{\circ}$ around the listening position. This recording represents a typical F-F

Table 1. Program items used in listening tests 1 (items 1-3) and 2 (items 4-6).

\begin{tabular}{|c|c|c|c|}
\hline No. & $\begin{array}{l}\text { Genre } \\
\text { Type }\end{array}$ & $\begin{array}{l}\text { Scene } \\
\text { Type }\end{array}$ & Description \\
\hline 1 & TV Sport & F-F & $\begin{array}{l}\text { Excerpt from Wimbledon (BBC catalog). Commentators and applause. Commentators panned } \\
\text { mid-way between L and } \mathrm{C} \text {, and } \mathrm{C} \text { and } \mathrm{R} \text {. Audience applause covers } 360^{\circ} \text {. }\end{array}$ \\
\hline 2 & Classical Music & F-B & $\begin{array}{l}\text { Excerpt from Johann Sebastian Bach - Concerto No.4 G-Major. Wide spatially-continuous front } \\
\text { stage including localizable instrument groups. Ambient surrounds with reverb from front stage. }\end{array}$ \\
\hline 3 & Rock/Pop Music & F-F & $\begin{array}{l}\text { Excerpt from Sheila Nicholls - Faith. Wide spatially-continuous front stage, including guitars, } \\
\text { bass, and drums. Main vocal in C. Harmony vocals, guitars, and drum cymbals in Ls and Rs. }\end{array}$ \\
\hline 4 & Jazz/Pop Music & F-B & $\begin{array}{l}\text { Excerpt from Max Neissendorfer and Barbara Mayr - I've Got My Love To Keep Me Warm. Live } \\
\text { music performance. Wide front stage. Ambience from room and/or audience in rear } \\
\text { loudspeakers. }\end{array}$ \\
\hline 5 & Dance Music & F-F & $\begin{array}{l}\text { Excerpt from Jean Michel Jarre - Chronology 6. Very immersive. Sources positioned all around } \\
\text { the listener. Some sources are moving. }\end{array}$ \\
\hline 6 & Film & F-B & $\begin{array}{l}\text { Excerpt from Jurassic Park } 2 \text { - The Lost World. Dialog in C. Ambience, sound effects and music } \\
\text { in L, R, Ls, and Rs. }\end{array}$ \\
\hline
\end{tabular}


Table 2. SAP groups used in listening tests 1 and 2

\begin{tabular}{cl}
\hline Group & \multicolumn{1}{c}{ Process type } \\
\hline 1 & Down-mixing from 5 channels \\
2 & Multichannel audio coding \\
3 & Altered loudspeaker locations \\
4 & Channel rearrangements \\
5 & Inter-channel level misalignment \\
6 & Inter-channel out-of-phase errors \\
7 & Channel removal \\
8 & Spectral filtering \\
9 & Inter-channel crosstalk \\
10 & Virtual surround algorithms \\
11 & Combinations of group 1-10 SAPs \\
12 & Anchor recordings \\
\hline
\end{tabular}

(foreground-foreground) ${ }^{2}$ scene type where each audio source is either close or clearly perceivable [36]. In comparison, program item 2 (classical music) is a classical recording with a different mix style, typical of many recordings from this genre, where the front three loudspeakers (i.e., left, center, and right) contain a wide spatially-continuous mix of the orchestra while the rear or surround loudspeakers contain ambient or reverberant energy. This recording represents a typical F-B (foreground-background) ${ }^{3}$ scene type.

\subsection{Spatial Audio Processes Evaluated}

Forty-eight different SAPs were chosen for evaluation to create a large number of stimuli, exhibiting a wide range of typical impairments to spatial quality. The selection was informed by discussions among the QESTRAL project group, by previous related studies $[12,37,38]$, and by the results of specific pilot studies [22]. The chosen SAPs can be divided into 12 groups (Table 2). Table 11 in the Appendix gives full descriptions.

It is possible that some SAPs may enhance, rather than degrade, spatial quality, but informal pilot evaluations by the authors indicated that, for the selections employed in this study, processed stimuli were never of a higher quality than the corresponding unprocessed reference. Within this study, therefore, the unprocessed reference stimuli were considered to be of optimal quality. If the results from the formal tests include processed stimuli rated at $100 \%$ quality then this will be revisited.

\subsection{Stimulus Loudness Equalization and Playback}

The stimuli (SAP and program item combinations) were loudness equalized using a listening panel. Each listener was asked to adjust playback gain to make all unprocessed reference stimuli equally loud and then to make each pro-

${ }^{2} \mathrm{~F}-\mathrm{F}$ (Foreground-Foreground) denotes Foreground program material (e.g., speech, musical sources) in the front loudspeakers and Foreground material in the rear.

${ }^{3}$ F-B (Foreground-Background) denotes Foreground material in the front loudspeakers and Background material (e.g., reverberation, applause) in the rear.

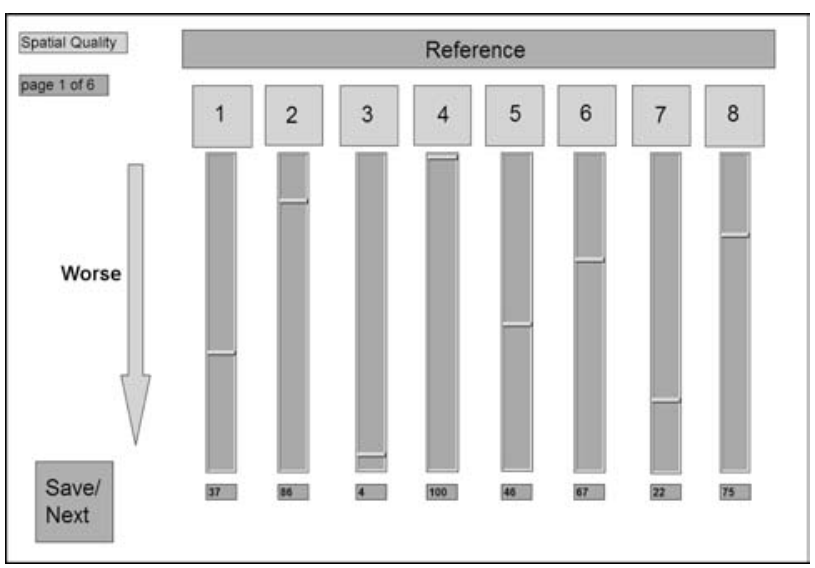

Fig. 2. Graphical user interface used in listening tests 1 and 2.

cessed stimulus equally loud to the corresponding original unprocessed reference. The means of the resulting gain adjustments were applied to the experiment stimuli. Overall playback gain was kept constant across all trials, having first been adjusted to provide a comfortable listening level. Thus, all stimuli were equally loud and measured $75-80 \mathrm{~dB}$ $\mathrm{L}_{\mathrm{AEQ}(1-3 \mathrm{mins})}$.

\subsection{Listening Test Method}

Pilot studies investigating the magnitude of perceptual differences between stimuli led to the choice of a multistimulus with hidden reference and anchors (MUSHRA) test method [39]. Listeners were presented with eight stimuli at a time and instructed to rate the spatial quality of each stimulus compared to an unprocessed reference program item. Listeners listened to the stimuli and recorded their responses using a graphical user interface (GUI) designed to reduce assessment scale biases inherent in listening tests (Fig. 2) [40][22]. The GUI was presented on a laptop situated at the listening position. The full instructions given to each listener, including a definition of spatial quality, are provided in the Appendix. It is acknowledged that listeners, although instructed to consider only spatial attributes, might also have considered timbral and other attributes. It will be important to take this possibility into consideration when the collected data are used to build a spatial quality model.

Quality ratings were recorded as integers from 0 to 100 . These are reported in later sections of this paper as percentages but it should be noted that they can only be considered as such within the context of the chosen scale end-points: the lowest anchor and the unprocessed reference. If a stimulus has a quality rating of $0 \%$ then this indicates that no other version of that program item presented in the experiment was perceived as having a lower quality; it does not indicate that quality could not possibly be lowered further. Similarly, if a stimulus has a quality rating of $100 \%$ then this indicates that no other version of that program item presented in the experiment was perceived as having a higher quality; it does not indicate that quality could not possibly be improved. 
Table 3. Anchor recordings used in listening tests 1 and 2

\begin{tabular}{ll}
\hline Anchor & \multicolumn{1}{c}{ Anchor description } \\
\hline Anchor recording A & $\begin{array}{c}\text { High anchor: unprocessed hidden } \\
\text { reference }\end{array}$ \\
Anchor recording B & $\begin{array}{l}\text { Mid anchor: audio codec }(80 \mathrm{kbs}) \\
\text { Anchor recording C } \\
\text { Low anchor: mono down-mix reproduced } \\
\end{array}$ \\
& $\begin{array}{l}\text { asymmetrically by the rear left } \\
\text { loudspeaker only }\end{array}$ \\
\hline
\end{tabular}

A full factorial experimental method was used so the listeners assessed every stimulus in every condition over four sessions at each listening position. The presentation order of the stimuli within each session was randomized. Each session consisted of the test and a repeat of the test and lasted approximately 30 minutes. Before commencing each session, listeners completed a familiarization trial to enable them to hear, and practice the assessment of, each stimulus. Fourteen listeners from the IoSR (Tonmeister and post-graduate students) with training in technical/critical listening and prior experience as listening test subjects, took part in listening test 1 and 17 took part in listening test 2. Due to the exploratory nature of the experiment, listeners were not specifically trained for it; it was important that they should interpret and rate the spatial quality of what they heard freely [25].

In accordance with the MUSHRA test method, three hidden indirect audio anchors, chosen to lie at the top, middle, and bottom of the test scale, were employed. These anchors were included on every test page in order to encourage listeners (without their knowledge) to use the rating scale more consistently from page to page and from test to test, and to reduce range equalization bias and centering bias [40]. They also allowed each listener's discrimination ability to be checked (see Secs. 2.1 and 3.1). The listeners were not informed of the anchors' presence. The anchors are detailed in Table 3. The high anchor was the unprocessed reference recording. The mid and low anchors were degraded using processes (representative of those used to generate the test stimuli) that a series of pilot studies [22] showed to produce appropriate levels of spatial degradation.

\section{LISTENING TEST 1 RESULTS AND DISCUSSION}

Listening test 1 compared SAP-degraded audio to unprocessed reference stimuli at both central and off-center listening positions. The SAPs employed in this test are indicated in Table 11 and were applied to program items 1-3. The following sections investigate the degree of perceived degradation and the factors affecting it. The intention is (i) to identify any data relating to unreliable listeners or a lack of inter-listener consensus, since these data would be unsuitable for inclusion in the database that will be used in the development of the quality evaluation system; and (ii) where there is consensus among reliable listeners, to learn more about the relationships between SAP, program item, listening position, and quality.
Table 4. ANOVA: significance and effect size of independent variables and interactions in listening test 1

\begin{tabular}{lccc}
\hline $\begin{array}{l}\text { Independent } \\
\text { variable }\end{array}$ & $\begin{array}{c}\text { Significance } \\
(p)\end{array}$ & $\begin{array}{c}\text { Partial-eta- } \\
\text { squared }\end{array}$ & F \\
\hline SAP & $<0.001$ & 0.891 & 1,865 \\
Listener $\times$ SAP & $<0.001$ & 0.413 & 12.29 \\
Program item $\times$ SAP & $<0.001$ & 0.234 & 34.77 \\
Listening position $\times$ SAP & $<0.001$ & 0.111 & 28.28 \\
\hline
\end{tabular}

\subsection{Data Screening}

Prior to results analysis each listener's responses were assessed, so that the unreliable data (i.e., data from a listener who lacked discrimination ability or consistency) could be removed. A listener's discrimination ability was established using a one-sided t-test to determine if their scores, throughout the listening test, for Anchor recording A were significantly different $(p<0.05$, degrees of freedom $=95)$ from the instructed value of 100; if they were not then that listener was deemed able to successfully identify that recording. A listener's consistency was assured if the RMS difference between their scoring of initial and repeat presentations of each SAP stimulus was less than $15 \%$. Although lower thresholds have been used in other studies [41] a higher threshold was chosen here due to the difficulty of the task.

The complete data sets of four of a total of 102 listeners were removed.

\subsection{Analysis of Variance}

After screening, the distributions of the SAP scores were assessed for normality using the Kolmogorov-Smirnov test (Field [42] cites this as being the most important test to guide choice of analysis technique). This showed $55 \%$ of the data to be normally distributed, indicating that parametric testing would be most suitable [ibid.]. A univariate Analysis of Variance (ANOVA) was conducted, with the independent variables included as fixed factors, to investigate the main effects of the independent test variables (SAP, listening position, program item, session, and listener), and their first-order interactions, on perceived spatial quality (dependent variable) $\left(r^{2}=0.908\right)$. The results for the variables of interest are presented in Table 4. Session was found to have no significant effect.

\subsection{Influence of Spatial Audio Process}

SAP had the largest effect on spatial quality ( $\mathrm{p}<0.001$, partial-eta-squared $=0.891$ ). Fig. 3 shows means and $95 \%$ confidence intervals for all SAPs (including the hidden anchors), averaged across both listening positions and all program items and listeners. The mean scores and confidence intervals for the SAPs cover the entire range of the test scale and have $95 \%$ confidence intervals narrower than 10 points $(10 \%)$ of the scale.

Overall, groups 1-10 predominantly created small (quality scores of $75 \%$ plus) to moderate (quality scores $50 \%$ to $75 \%$ ) impairments to the perceived spatial quality. However, some SAPs in groups 1 (downmixing), 9 (crosstalk), 


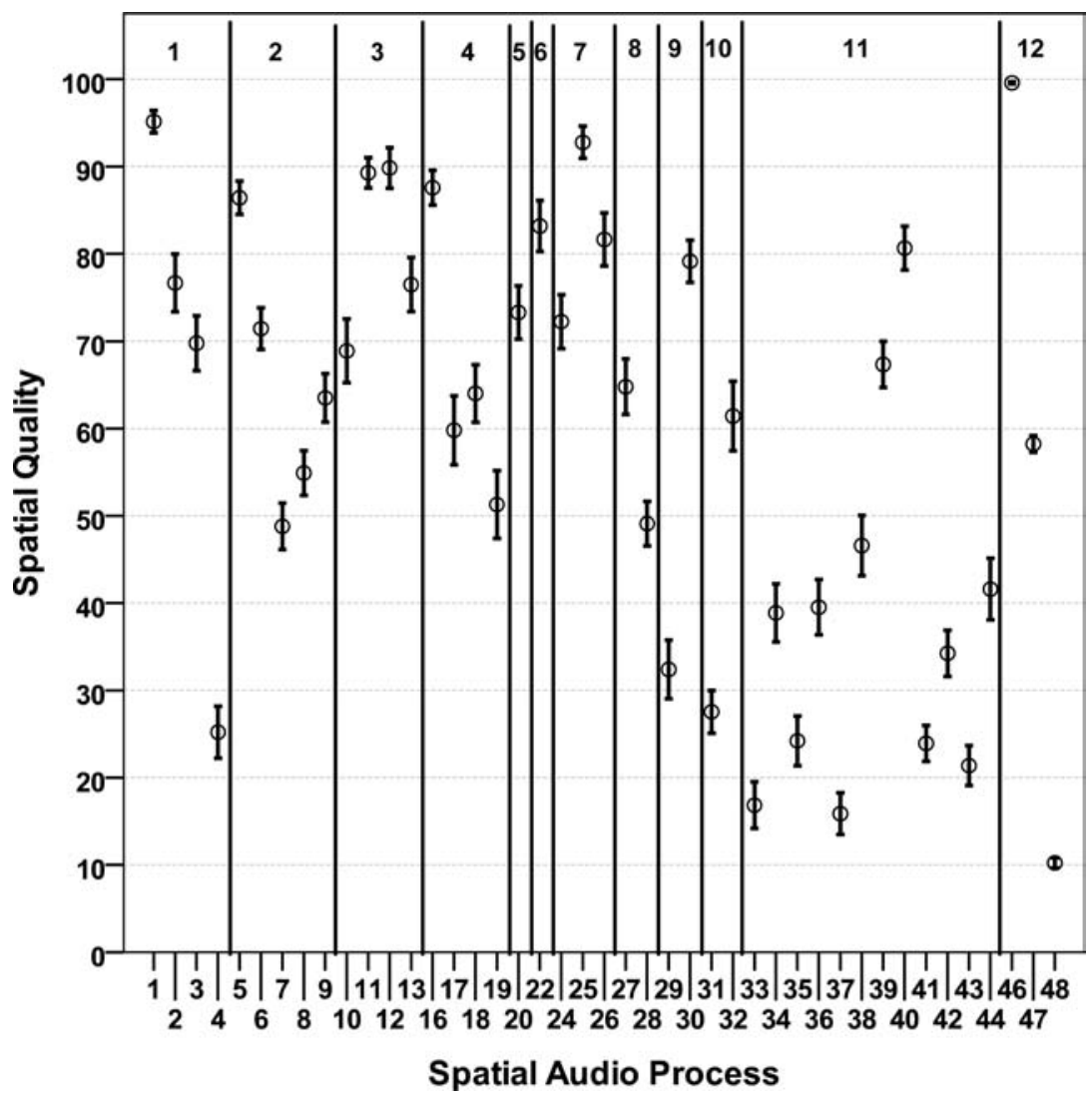

Fig. 3. Mean spatial quality scores for each SAP in listening test 1, averaged across program item type, listening position, and listener.

and 10 (virtual surround) produced large changes to inter-channel relationships (sometimes to the extent that the resulting auditory image was perceived as being inhead, as with SAPs 29 and 37 for example) and reduced quality severely (quality scores less than 50\%). Many SAPs in group 11 (combinations of 1-10) also created severe impairments. This is not surprising as these SAPs compound the degradation created by two different processes.

In group 2 (multichannel audio coding), only the lowest bit-rate process achieved a mean score of less than $50 \%$ (and even then not significantly so). The SAPs in groups 3 (altered loudspeaker locations), 4 (channel rearrangements), and 8 (spectral filtering) also reduced quality by small to moderate amounts, again with no mean scores significantly less than $50 \%$. No group 3 SAP produced a mean quality score significantly below $70 \%$.

The smallest impairment to spatial quality was created by SAP 1 (3/1 downmix) but, in general, groups 5 (interchannel level misalignment), 6 (inter-channel out-of-phase errors), and 7 (channel removal) seemed least capable of degrading quality (with no score significantly below $75 \%)$.

The anchor recordings (group 12) were all scored in their expected locations. Anchor recording A, the unprocessed reference, was scored at $100 \%$. (NB. The confidence intervals for this group are small due to the anchors appearing on every test page and therefore being assessed many more times than the other SAPs.)

\subsection{Influence of Listener}

The interaction between listener and SAP had the second largest effect on perceived spatial quality ( $p<0.001$, partial-eta-squared $=0.413$ ), and this suggests that there was a difference in opinion or lack of consensus between listeners with respect to the qualities of certain stimuli. Further experimental work might provide insights into the reason(s) for this lack of consensus (listener reliability was validated in Sec. 2.1 and so this will not be a factor) but, for the purpose of the analysis presented in this paper, it will be sufficient to identify the stimuli concerned. A number of statistical and visual analysis techniques_-including the Kolmogorov-Smirnov test, modality, standard deviation, data range, and kurtosis (z-score) test-were used to identify stimuli which have scores exhibiting a multimodal, wide or platykurtic distribution (Fig. 4a); for comparison, a stimulus with scores having a statistically normal distribution and reliable average is depicted in Fig. $4 \mathrm{~b}$. Score averages for stimuli producing platykurtic distributions will not be meaningful or reliable; therefore the effects of the corresponding SAPs on spatial quality cannot be defined. Consequently, results relating to stimuli where this effect is observed-where the standard deviation of the data distribution is greater than 20 , the data range is greater than $75 \%$ and kurtosis score is greater than -1 - should not feed into the development of a quality evaluation system. Combinations of program item, SAP, and listening position identified as having unreliable average scores are listed in Table 5, which shows that $11 \%$ of the data should be 

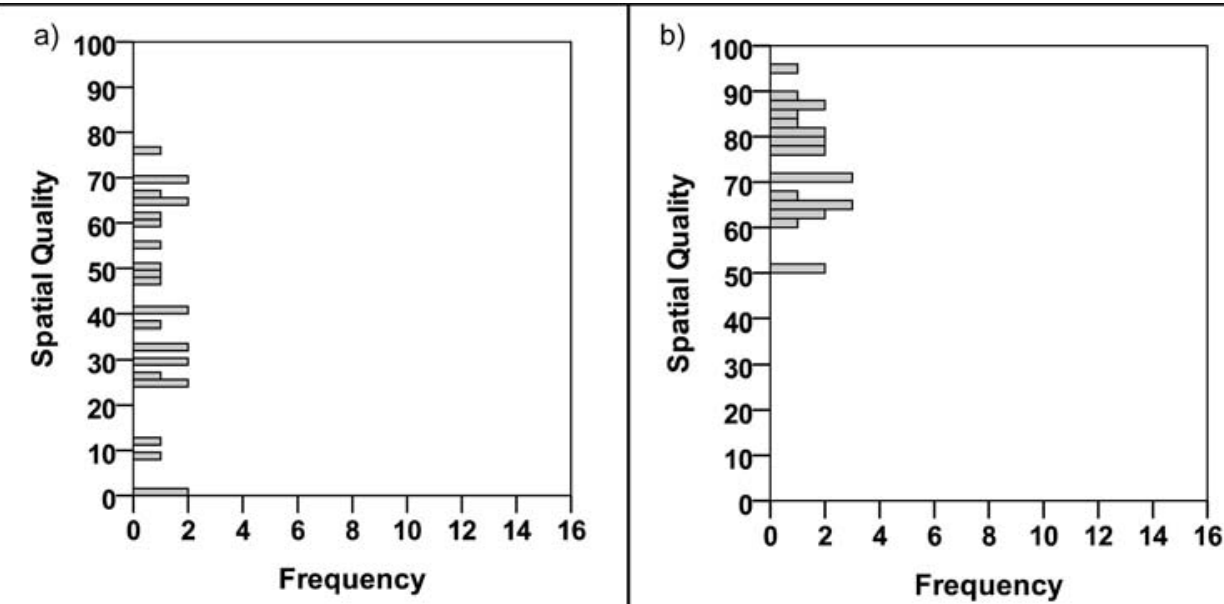

Fig. 4. (a) Stimulus producing a platykurtic score distribution. (b) Stimulus producing a statistically normal score distribution. (Distributions categorized by tests described in Sec. 2.4.)

Table 5. Stimuli producing unreliable average scores in listening test 1 (refer to Table 11 for descriptions)

\begin{tabular}{lcl}
\hline $\begin{array}{l}\text { Listening } \\
\text { position }\end{array}$ & Program item & \multicolumn{1}{c}{ Spatial audio process } \\
\hline 1 & 1 & $7,32,33$ \\
& 2 & $7,17,19,22,27,34,36,38,44$ \\
2 & 3 & $6,7,19,32,44$ \\
& 1 & $18,19,20$ \\
& 2 & $4,19,27,29$ \\
& 3 & $4,8,19,27,29$ \\
\hline
\end{tabular}

Table 6. SAPs producing significantly different scores for different program items in listening test 1 (refer to Table 11 for descriptions)

\begin{tabular}{lc}
$\begin{array}{l}\text { Listening } \\
\text { position }\end{array}$ & Spatial audio process \\
\hline 1 & $1,2,3,5,9,10,11,12,13,16,17,18,19,22,24$, \\
& $25,26,32,33,34,38,39,40,42,44,46,47$ \\
2 & $1,2,3,5,9,10,11,12,13,16,17,19,22,24,26$, \\
& $30,32,34,39,40,41,42,43,46,47$ \\
\hline
\end{tabular}

removed. In cases where the distribution was non-normal but leptokurtic, and the other tests had been passed, the median value will be taken to be a reliable average score.

\subsection{Influence of Program Item}

The interaction of program item type with SAP had a significant effect on perceived spatial quality $(p<0.001$, partial-eta-squared $=0.234$ ). This indicates that certain SAPs degraded spatial quality more for some program items than for others. Therefore, in the development of a spatial quality evaluation system, SAP scores obtained from one program item should ideally be considered separately from those obtained from another. A one-way ANOVA using program item as the factor was used to determine which SAPs exhibited this effect $(p<0.05)$, and these are listed in Table 6.
In many cases the difference in the perceived spatial quality between program items can be accounted for by differences in spatial scene-type. For example, a far smaller impairment resulted when a 3.0 downmix was applied to program item 2 (classical music) than when it was applied to items 1 (TV/sport) and 3 (rock/pop). This is because the rear channels of item 2 contained only background ambient or reverberant information, which was included to enhance the spaciousness or presence in the recording. This background content was diffuse and not very localizable and so down-mixing it into the front channels did not create an overly degrading impairment. This is different from program items 1 and 3 whose rear channels contained clearly identifiable foreground sources. The effect occurs at both listening positions as shown in Fig. 5. A similar observation was made in a study conducted by Zieliński et al. [36].

Other aspects of audio content may also have been factors. For example, when the channel order of program item 1 was changed randomly, a lesser impairment resulted than when the same process was applied to item 2 or item 3 . This can be explained by the fact that most of the channels in program item 1 contain audience applause whose location in the audio scene is unimportant. Hence the channels can be re-routed at random without significant impairment to the overall spatial quality (nevertheless, a slight impairment was created because channels containing the commentators' voices were also re-routed). Conversely, re-routing channels in program items 2 and 3 destroyed the intended audio image. Again this effect occurs at both listening positions, as shown in Fig. 6.

\subsection{Influence of Listening Position}

The interaction of listening position with SAP had a significant effect on perceived spatial quality $(p<0.001$, partial-eta-squared $=0.111)$. This suggests that certain SAPs impaired spatial quality more when auditioned at one listening position than when auditioned at the other. Therefore, in the development of a spatial quality evaluation system, as with scores for different program items, SAP 


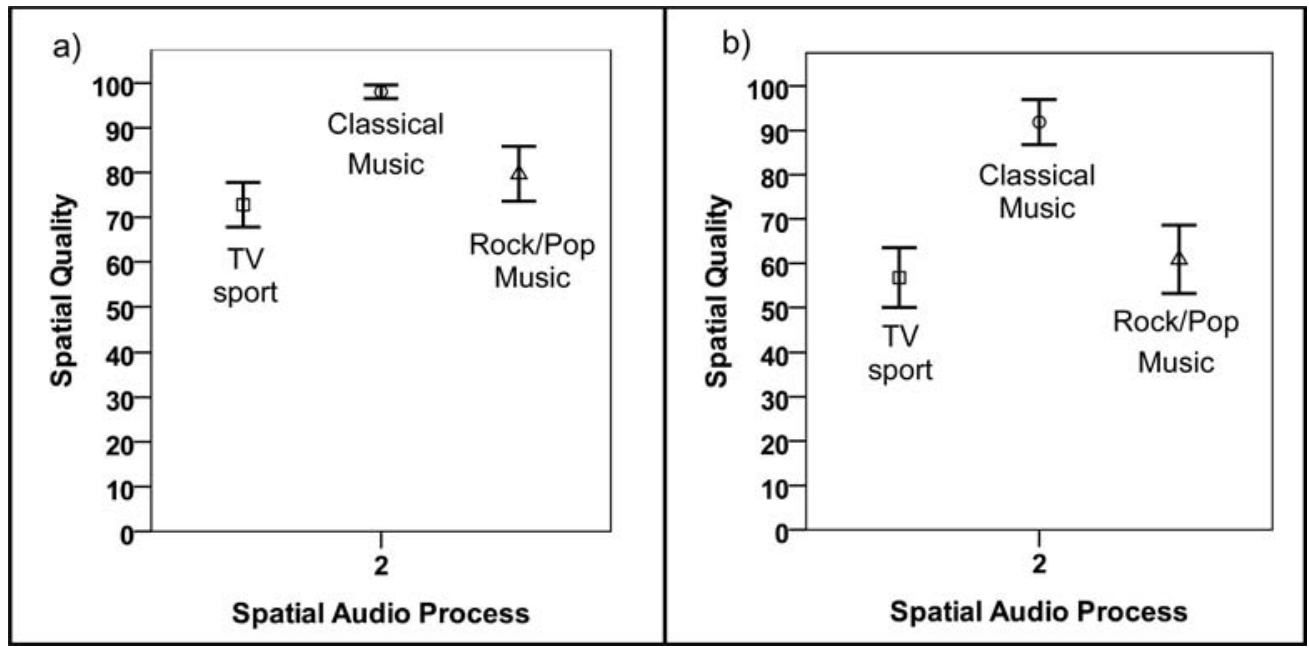

Fig. 5. The mean quality degradation resulting from a downmixing process (SAP 2) is greater for TV/sport and rock/pop music program items than for classical music at both (a) LP1 and (b) LP2.

Table 7. SAPs producing significantly different scores for different program items in listening test 1 (refer to Table 11 for descriptions)

\begin{tabular}{lc}
\hline $\begin{array}{l}\text { Program } \\
\text { item }\end{array}$ & \multicolumn{1}{c}{ Spatial audio process } \\
\hline 1 & $1,2,12,13,19,20,22,25,29,30,31,33,34,38$, \\
& $39,30,44,47$ \\
2 & $1,2,5,12,13,19,22,24,26,29,31,42,44,47$ \\
3 & $2,3,12,13,17,24,29,31,33,34,35,36,38,39$, \\
& $40,44,47$ \\
\hline
\end{tabular}

scores at LP1 should ideally be considered separately from those at LP2. A one-way ANOVA with listening position as the factor was used to determine which stimuli exhibited this effect $(p<0.05)$, and these are listed in Table 7 .

The effect can be explained by the physical location change between LP1 and LP2 altering the audio informa- tion that listeners received. For example, when the rear loudspeakers were misplaced to $-90^{\circ}$ and $90^{\circ}$ respectively (SAP 12), only a small impairment to spatial quality resulted at LP1; this fits with the Minimum Audible Angle theory that predicts the inability of the human auditory system to accurately locate sound sources positioned in an area around each ear (at approximately $\pm 90^{\circ}$ ) [43]. However, LP2 is closer to the right surround loudspeaker and so the misplacement of the rear loudspeakers was likely to have been much more obvious, making the impairment greater and the SAP score lower. This effect is observed for all three program item types, as shown in Fig. 7.

\section{LISTENING TEST 2 RESULTS AND DISCUSSION}

Listening test 2 compared SAP-degraded audio at central and off-center listening positions to centrally-auditioned

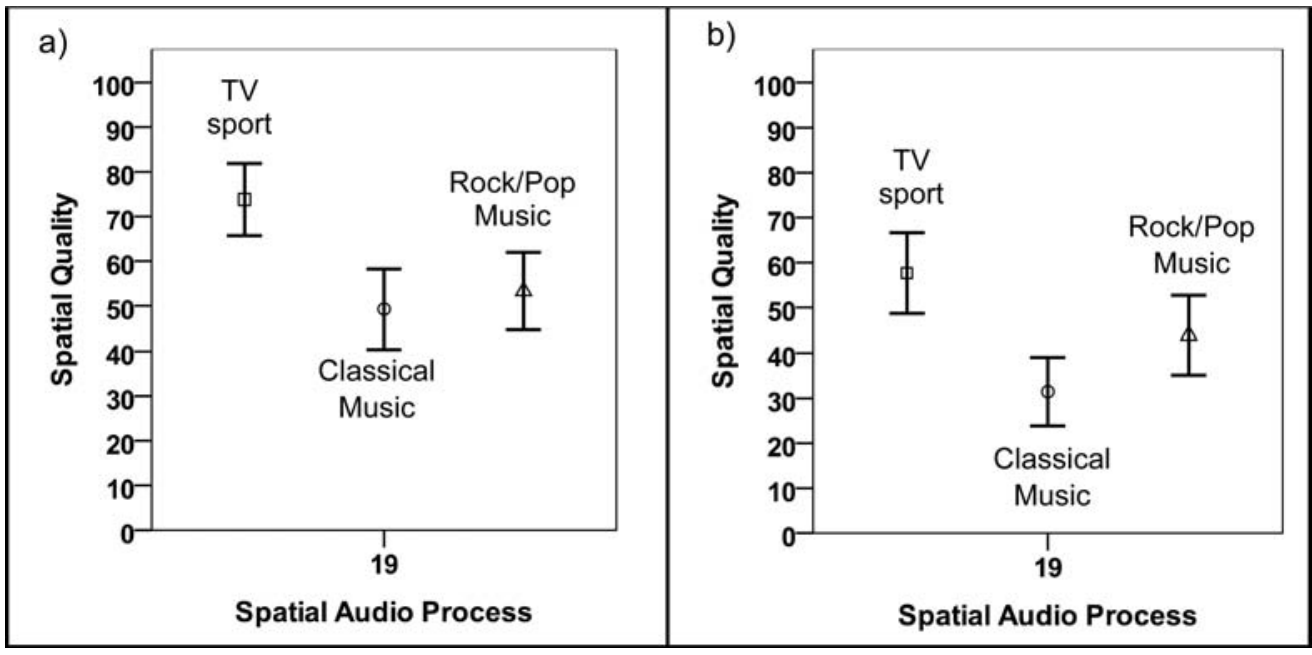

Fig. 6. The mean quality degradation resulting from a channel-swapping process (SAP 17) is greater for classical music and rock/pop program items than for TV/sport at both (a) LP1 and (b) LP2. 


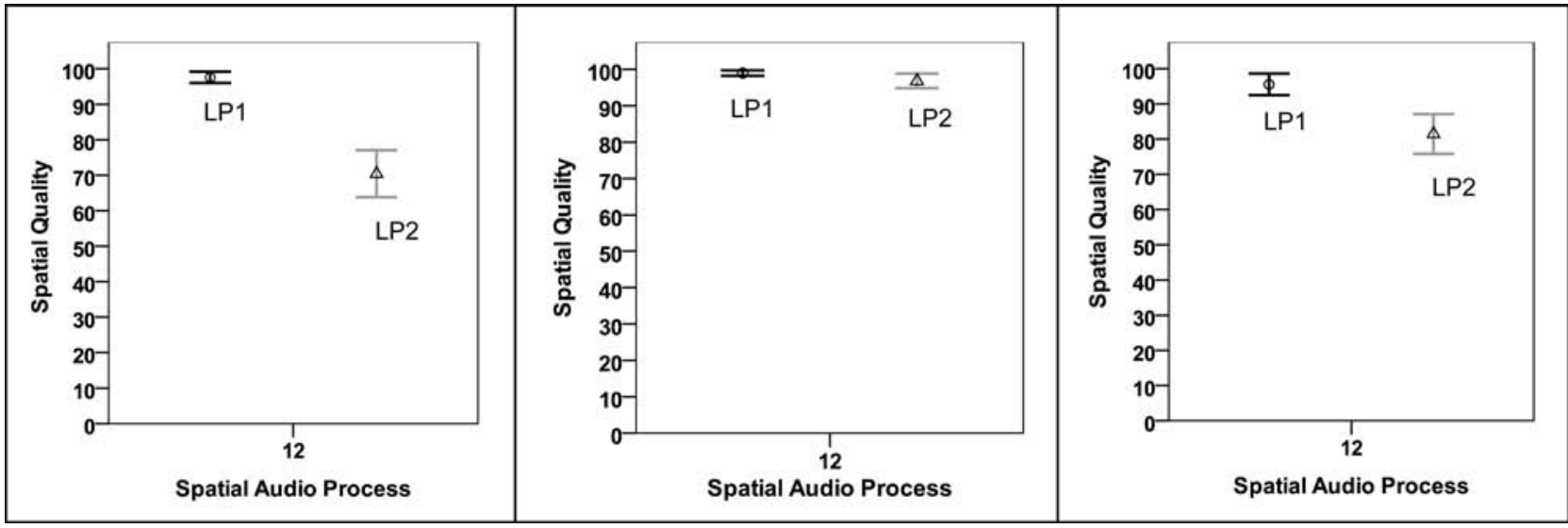

Fig. 7. The mean quality degradation resulting from a loudspeaker mis-positioning (SAP 12) is greater at LP2 than at LP1, for program items (a) 1, (b) 2, and (c) 3 .

unprocessed reference stimuli. The SAPs employed in this test are indicated in Table 11 and were applied to program items 4-6. The following sections investigate the degree of perceived degradation and the factors affecting it. As with the test 1 analysis, the intention is (i) to identify any data relating to unreliable listeners or a lack of inter-listener consensus, since these data would be unsuitable for inclusion in the database that will be used in the development of the quality evaluation system; and (ii) where there is consensus among reliable listeners, to learn more about the relationships between SAP, program item, listening position, and quality.

\subsection{Data Screening}

Prior to analysis each listener's responses were assessed in the same manner as listening test 1 so that the most reliable data could be selected for investigation. The complete data sets of 13 of a total of 68 listeners were removed.

\subsection{Analysis of Variance}

After screening, the distributions of the SAP scores were assessed for normality using the Kolmogorov-Smirnov test. This showed $65 \%$ of the data to be normally distributed, meaning that parametric testing could be employed. A univariate ANOVA was conducted, with the independent variables included as fixed factors, to investigate the main effects of the independent test variables (SAP, listening position, program item, session, and listener), and their firstorder interactions, on perceived spatial quality (dependent variable) $\left(r^{2}=0.861\right)$. The results for the variables of interest are presented in Table 8. Session was again found to have no significant effect.

\subsection{Influence of Spatial Audio Process and Listening Position}

As with listening test 1, SAP had the largest effect on spatial quality $(p<0.001$, partial-eta-squared $=0.682)$ in listening test 2 . Fig. 8 shows means and $95 \%$ confidence intervals for all SAPs (including the anchors) for both LP1
Table 8. ANOVA: significance and effect size of independent variables and interactions in listening test 2

\begin{tabular}{lccc}
\hline $\begin{array}{l}\text { Independent } \\
\text { variable }\end{array}$ & $\begin{array}{c}\text { Significance } \\
(p)\end{array}$ & $\begin{array}{c}\text { Partial-eta- } \\
\text { squared }\end{array}$ & F \\
\hline SAP & $<0.001$ & 0.682 & 466.9 \\
Listener $\times$ SAP & $<0.001$ & 0.433 & 12.26 \\
Program item $\times$ SAP & $<0.001$ & 0.128 & 16.02 \\
Listening Position & $<0.001$ & 0.085 & 444.0 \\
\hline
\end{tabular}

and LP2, averaged across all program items and listeners. As with the test 1 results, the mean scores and confidence intervals for the evaluated spatial audio processes cover the entire range of the test scale and in all but a few cases have $95 \%$ confidence intervals narrower than 10 points $(10 \%)$ of the scale.

Trends in terms of which groups exhibited small, moderate, and severe quality impairments are the same as those observed in listening test 1: again groups 1-8 predominantly showed small to moderate quality impairments but with some SAPs in group 1 (SAP 4: 1.0 downmix), group 9 (SAP 29: 1.0 downmix in all channels), and group 11 (SAP 37: 1.0 downmix $+500 \mathrm{HPF}$ on all channels) reducing quality severely; groups $2,3,4$, and 8 reduced quality by small to moderate amounts; groups 5,6 , and 7 exhibited only small impairments; and the anchors (group 12) were all scored in their intended locations.

Separating the scores for LP1 (circles) and LP2 (triangles) illustrates how spatial quality was further impaired when listening off-center. Similar overall scoring trends are observable in the LP1 and LP2 data. However, the range of the scores for LP2 is compressed into the lower part of the test scale. The difference in perceived quality between LP1 and LP2 for the highest quality SAPs is as much as $30 \%$ (e.g., SAP 1, circled), whereas the difference between LP1 and LP2 scores for the lowest-rated SAPs is less than $5 \%$ and is statistically not significant (e.g., SAP 37, also circled). This smaller difference for the lowest-rated SAPs suggests that the impairment to spatial quality resulting from these processes is already so severe that a shift 


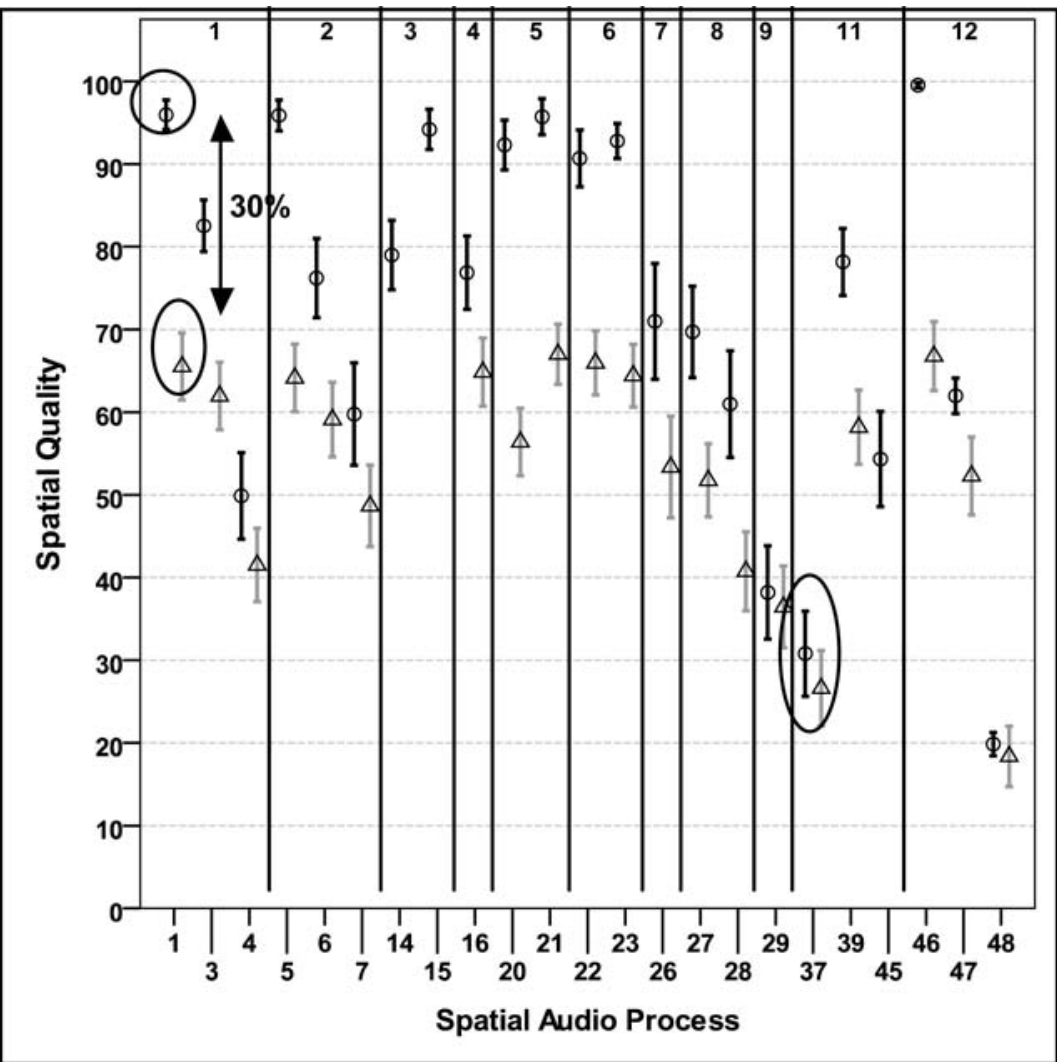

Fig. 8. Mean spatial quality ratings for each SAP in listening test 2, averaged across program item type and listener; note the compression of the rating range at LP2 (triangles) compared to that at LP1 (circles).

Table 9. Stimuli producing unreliable average scores in listening test 2 (refer to Table 11 for descriptions)

\begin{tabular}{lcc}
\hline Listening position & Program item & Spatial audio process \\
\hline 1 & 4 & $4,6,7,27,28,29,45$ \\
& 5 & $27,29,45$ \\
2 & 6 & $4,26,27,28,29,37$ \\
& 4 & 4,28 \\
& 5 & $4,16,20,28$ \\
& 6 & $26,29,37$ \\
\hline
\end{tabular}

in the listening position is unable to produce any further degradation.

\subsection{Influence of Listener}

As in listening test 1 , listeners' scores reveal a difference in opinion and a lack of consensus for certain stimuli $(p<0.001$, partial-eta-squared $=0.433)$. This was investigated further (as in listening test 1) to determine that $19 \%$ of stimuli should be treated as having unreliable average scores. Table 9 summarizes the results of this analysis.

\subsection{Influence of Program Item}

The interaction of program item type with process is again shown to have a significant effect on perceived spatial quality $(p<0.001$, partial-eta-squared $=0.128$ ). A oneway ANOVA using program item as the factor was used to determine which stimuli exhibited this effect. The list of
Table 10. SAPs producing significantly different scores for different program items in listening test 2 (refer to Table 11 for descriptions)

\begin{tabular}{ll}
\hline Listening position & \multicolumn{1}{c}{ Spatial audio process } \\
\hline 1 & $1,3,6,14,15,22,26,39$ \\
2 & $6,20,26,39$ \\
\hline
\end{tabular}

SAPs where this test was found to be statistically significant $(p<0.05)$ is given in Table 10.

\section{SUMMARY AND CONCLUSIONS}

SAPs commonly encountered in consumer audio reproduction systems are known to generate a range of impairments to spatial quality. By way of two listening tests, this paper investigated the degree of degradation of the spatial quality of six 5-channel audio recordings, resulting from 48 such SAPs, and the influences of listening position and source material on that degradation, and built a qualityannotated database of processed and unprocessed program items.

Choice of SAP has a large effect on degradation degree. SAPs producing large changes to inter-channel relationships (downmix and virtual surround algorithms and the introduction of high levels of crosstalk) can reduce quality severely (quality scores significantly $<50 \%$ ), as can combinations of multiple SAPs. Conversely, inter-channel level 
and phase misalignment, and channel removal, seem able to degrade quality only slightly (no score significantly below 75\%). Other SAPs (lossy coding, moved or missing loudspeakers and spectral filtering) fall between these two extremes (no score significantly below 50\%). Future development of a spatial audio quality evaluation system must therefore take into account the effects of a wide range of SAPs.

The effect of the interaction between listener and SAP can also be large (although, in this study, less than that of SAP alone). Although the majority (86\%) of the collected data show inter-listener consensus, it appears that for some stimuli there is disagreement between listeners with regard to the degree of degradation present. Means of data relating to such stimuli cannot be treated as reliable and so should not feed into the development of a future spatial audio quality evaluation system. For the majority of stimuli evaluated, however, there is agreement between listeners and so score averages relating to the bulk of the data collected can be used.

There can also be a noticeable effect from the interaction between SAP and program item. This effect is observable for some SAPs more than others. SAPs that alter the playback positions of one or more channels (e.g., downmixing algorithms, repositioned loudspeakers, channel-order changes) seem particularly susceptible to this interaction, which in many cases can be accounted for by variations in spatial scene type from item to item (e.g., whether or not the surround channels contain distinct sound sources). The size and frequency of this interaction effect means that, in the development of a spatial quality evaluation system, SAP scores obtained from one program item should ideally be considered separately from those obtained from another. If a single particularly revealing program item is sought for SAP quality testing then an item having foreground sources in every channel should be chosen.

Listening position is important in two respects. First, interaction effects are observable: listening position can affect the degree of perceived quality degradation resulting from a SAP. This is particularly evident when a primary effect of a SAP is to alter the output or position of a loudspeaker that is closer to the listener in an off-center listening position. Therefore, as with program items, in follow-on work SAP scores obtained at one listening position should ideally be considered separately from those obtained at another. Second, combining off-center listening with another SAP can reduce quality by as much as $30 \%$ compared to auditioning that SAP centrally, but the additional deleterious effects of off-center listening lessen (to insignificance) when a severely degrading SAP is used.

Taken together these findings, and the quality-annotated database, can guide the development of a regression model of perceived overall spatial audio quality, incorporating previously developed spatially relevant feature-extraction algorithms. A quality evaluation system based on such a model will have the potential to provide an indication of likely perceived audio quality where human assessment would be impractical or impossible. The development of such a model will be documented in a follow-up paper.

\section{ACKNOWLEDGMENTS}

This research was completed as a part of the QESTRAL Project (Engineering and Physical Sciences Research Council EP/D041244/1), a collaboration between the University of Surrey (UK), Bang \& Olufsen (Denmark), and BBC Research and Development (UK).

\section{REFERENCES}

[1] F. Rumsey Spatial Audio (Focal Press, 2001).

[2] G. A. Soulodre, M. C. Lavoie, S. G. Norcross, "Objective Measures of Listener Envelopment in Multichannel Surround Systems," J. Audio Eng. Soc., vol. 51, pp. 826840 (2003 Sep.).

[3] M. Davis "History of Spatial Coding," J. Audio Eng. Soc., vol. 51, pp. 554-569 (2003 June).

[4] F. Rumsey "Spatial Audio-Eighty Years after Blumlein,” J. Audio Eng. Soc., vol. 59, pp. 57-65 (2011 Jan./Feb.).

[5] F. Rumsey “Audio for Games," J. Audio Eng. Soc., vol. 59, pp. 341-345 (2011 May).

[6] F. Rumsey, "Spatial Quality Evaluation for Reproduced Sound: Terminology, Meaning, and a Scene-Based Paradigm,” J. Audio Eng. Soc., vol. 50. pp. 651-666 (2002 Sep.).

[7] J. A. Belloch, M. Ferrer, A. Gonzalez, F. J. MartinezZaldivar and A. M. Vidal, "Headphone-Based Virtual Spatialization of Sound with a GPU Accelerator," J. Audio Eng. Soc., vol. 61, pp. 546-561 (2013 Jul./Aug.).

[8] BBC, "Surround Sound," http://www.bbc.co.uk/ bbchd/what_is_hd.shtml [Accessed 11/08/10] (2009).

[9] BSkyB Ltd., "Experience more with Sky+HD," http://packages.sky.com/hd/?DCMP=ILC-SkyCOM_HD [Accessed 11/08/10] (2009).

[10] P. Marins, F. Rumsey and S. Zieliński, "Unravelling the Relationship between Basic Audio Quality and Fidelity Attributes in Low Bit-Rate Multichannel Audio Codecs," presented at the 124th Convention of the Audio Engineering Society (2008 May), convention paper 7335.

[11] ITU-R BS.775-1, "Multi-channel Stereophonic Sound System with and without Accompanying Picture," International Telecommunication Union recommendation (1992-1994).

[12] S. Zieliński, F. Rumsey, and S. Bech "Comparison of Quality Degradation Effects Caused by Limitation of Bandwidth and by Down-mix Algorithms in Consumer Multichannel Audio Delivery Systems," presented at the 114th Convention of the Audio Engineering Society (2003 Mar.), convention paper 5802 .

[13] F. Rumsey "Spatial Audio Processing," J. Audio Eng. Soc., vol. 61, pp. 474-478 (2013 June).

[14] S. Bech and N. Zacharov Perceptual Audio Evaluation: Theory, Method and Application (John Wiley \& Sons Ltd., West Sussex, UK, 2006).

[15] ITU-R BS.1387, "Method for Objective Measurements of Perceived Audio Quality," International Telecommunication Union recommendation (2001).

[16] J. Liebetrau, T. Sporer, S. Kämpf, and S. Schneider, "Standardization of PEAQ-MC: Extension of ITU-R 
BS.1387-1 to Multichannel Audio," AES 40th International Conference: Spatial Audio (2010 Oct.), conference paper P-3.

[17] I. Choi, B. G. Shinn-Cunningham, S. B Chon, and K. Sung, "Objective Measurement of Perceived Auditory Quality in Multichannel Audio Compression Coding Systems," J. Audio Eng. Soc., vol. 56, pp. 3-17 (2008 Jan./ Feb.).

[18] S. George "Objective Models for Predicting Selected Multichannel Audio Quality Attributes," Ph.D. Thesis, Institute of Sound Recording, University of Surrey (2009).

[19] J-H. Seo, I. Choi, S. B. Chon, and K-M Sung "Improved Prediction of Multichannel Audio Quality by the Use of Envelope ITD of High Frequency Sounds," AES 38th International Conference: Sound Quality Evaluation (2010 June), conference paper 5-1.

[20] M. Dewhirst, "Modelling Perceived Spatial Attributes of Reproduced Sound," Ph.D. Thesis, Institute of Sound Recording, University of Surrey, http://epubs.surrey. ac.uk/2081/ (2008).

[21] P. J. B Jackson, M. Dewhirst, R. Conetta, F. Rumsey, S. Zieliński, S. Bech, D. Meares and S. George, "QESTRAL (Part 3): System and Metrics for Spatial Quality Prediction," presented at the 125th Convention of the Audio Engineering Society (2008 Oct.), convention paper 7597.

[22] R. Conetta "Towards the Automatic Assessment of Spatial Quality in the Reproduced Sound Environment," Ph.D. Thesis, Institute of Sound Recording, University of Surrey, http://epubs.surrey.ac.uk/39628/ (2011).

[23] T. Letowski, "Sound Quality Assessment: Cardinal Concepts," presented at the 87th Convention of the Audio Engineering Society (1989 Oct.), convention paper 2825.

[24] J. Berg "The Contrasting and Conflicting Definitions of Envelopment," presented at 126th Convention of the Audio Engineering Society (2009 May), convention paper 7808 .

[25] J. Berg and F. Rumsey "Identification of Quality Attributes of Spatial Audio by Repertory Grid Technique," J. Audio Eng. Soc., vol. 54, pp. 365-379 (2006 May).

[26] S. Choisel and F. Wickelmaier "Extraction of Auditory Features and Elicitation of Attributes for the Assessment of Multichannel Reproduced Sound" presented at the 118th Convention of the Audio Engineering Society (2005 May), convention paper 6369.

[27] K. Koivuniemi and N. Zacharov, "Unravelling the Perception of Spatial Sound Reproduction: Language Development, Verbal Protocol Analysis and Listener Training," presented at the 111th Convention of the Audio Engineering Society (2001 Nov.-Dec.), convention paper 5424.

[28] F. Rumsey, S. Zieliński, R. Kassier, and S. Bech "Relationships between Experienced Listener Ratings of Multichannel Audio Quality and Naïve Listener Preferences," J. Acoust. Soc. Am., vol. 117, no. 6, pp. 3832-3840 (2005).

[29] N. Zacharov and K. Koivuniemi, "Unraveling the Perception of Spatial Sound Reproduction: Techniques and Experimental Design,” AES 19th International Conference:
Surround Sound-Techniques, Technology, and Perception (2001 June), conference paper number 1929.

[30] N. Zacharov and K. Koivuniemi, "Unraveling the Perception of Spatial Sound Reproduction: Analysis and External Preference Mapping," presented at the 111th Convention of the Audio Engineering Society (2001 Sep.), convention paper 5423 .

[31] H. A. M. Clark, G. F. Dutton, and P. B. Vanderlyn, "The 'Stereosonic' Recording and Reproducing System: A Two-Channel System for Domestic Tape Records," J. Audio Eng. Soc., vol. 6, pp. 102-117 (1958 Apr.).

[32] J. Eargle "An Analysis of Some Off-Axis Stereo Localization Problems," presented at the 81st Convention of the Audio Engineering Society (1986 Nov.), convention paper 2390.

[33] S. Merchel and S. Groth "Adaptively Adjusting the Stereophonic Sweet Spot to the Listener's Position," J. Audio Eng. Soc., vol. 58, pp. 809-817 (2010 Oct.).

[34] ITU-R BS.1116-1, "Methods for the Subjective Assessment of Small Impairments in Audio Systems including Multichannel Sound Systems," International Telecommunication Union recommendation (1997).

[35] Bang \& Olufsen, "Beolab 3 specifications," http://www.bang-olufsen.com/en/sound/loudspeakers/ beolab-3 [Accessed 06/09/14] (2011).

[36] S. Zieliński, F. Rumsey, S. Bech, and R. Kassier "Effects of Down-Mix Algorithms on Quality of Surround Sound," J. Audio Eng. Soc., vol. 51, pp. 780-798 (2003 Sep.).

[37] S. Zieliński, F. Rumsey, and S. Bech "Effects of Bandwidth Limitation on Audio Quality in Consumer Multichannel Audiovisual Delivery Systems," J. Audio Eng. Soc., vol. 51, pp.475-501 (2003 June).

[38] S. Zieliński, F. Rumsey, S. Bech, and R. Kassier "Comparison of Basic Audio Quality and Timbral and Spatial Fidelity Changes Caused by Limitation of Bandwidth and by Downmix Algorithms in 5.1 Surround Audio Systems," J. Audio Eng. Soc., vol. 53, pp.174-192 (2005 Mar.).

[39] ITU-R BS.1534, "Method for the Subjective Assessment of Intermediate Audio Quality," International Telecommunication Union recommendation (2001).

[40] S. Zieliński, F. Rumsey and S. Bech "On Some Biases Encountered in Modern Audio Quality Listening Tests-A Review," J. Audio Eng. Soc., vol. 56, pp. 427451 (2008 June).

[41] F. Rumsey "Subjective Assessment of the Spatial Attributes of Reproduced Sound" AES 15th International Conference: Audio, Acoustics \& Small Spaces (1998 Oct.), conference paper 15-012.

[42] A. Field Discovering Statistics Using SPSS, 2nd Ed. (SAGE Publications Ltd., UK, 2005).

[43] B. C. J. Moore An Introduction to the Psychology of Hearing, 5th Ed. (Academic Press, UK, 2003).

\section{APPENDIX}

The instructions given to each listener before commencing listening test 1 and 2 are presented followed by Table 11 that lists SAP descriptions and groupings. 


\section{Listener Instructions}

Thank you for participating in this experiment.

Please read the instructions below.

\section{Description of subject task and scale for spatial quality score}

You are asked to compare a number of spatial sound recordings, which have been processed or degraded in various ways, with an unprocessed original reference recording. You are asked to rate the spatial quality of the processed items. A spatial quality scale is a hybrid scale that is primarily a fidelity evaluation (one measuring the degree of similarity to the reference). However it also enables you to give an opinion about the extent to which any differences are inappropriate, unpleasant or annoying. In other words, which affect your opinion of the quality of the spatial reproduction compared with the reference. So, for example, if you can hear a change in the spatial reproduction compared with the reference but it doesn't make much difference to your overall opinion about the spatial quality, you should rate it towards the top of the scale. On the other hand, if the spatial change is very pronounced and you consider it to be annoying, unpleasant or inappropriate, you should probably rate it towards the bottom of the scale. In the middle should go items that have clearly noticeable changes in the spatial reproduction and that are only moderately annoying, unpleasant or inappropriate. It is up to you how you interpret these terms but the aim is to come up with an overall evaluation of your opinion of the spatial quality of the processed items compared with the reference. It comes down to a judgement about how acceptable the impairments of the test items are when you know what the original recording (the reference) should sound like.

In order to avoid any potential biasing effects of verbal labels with particular meanings at intervals on the scale, the scale you will use simply has a magnitude and an overall direction labelled 'worse'. Any item rated at the top of the scale should be considered as identical to the reference. Try to use the whole scale, rating the worst items in the test at the bottom of the scale and the best ones at the top. Try to ignore any changes in quality that are not spatial, unless they directly affect spatial attributes.

The following are examples of changes in spatial attributes that you may hear and may incorporate in your overall evaluation (in no particular order of importance, and not meant to exclude any others you may hear):

Changes in location

Changes in rotation or skew of the spatial scene

Changes in width

Changes in focus, precision of location or diffuseness

Changes in stability or movement

Changes in distance or depth

Changes in envelopment (the degree to which you feel immersed by sound)

Changes in continuity (appearance of 'holes' or gaps in the spatial scene)

Changes in perceived spaciousness (the perceived size of the background spatial scene, usually implied by reverberation, reflections or other diffuse cues)

Other unnatural or unpleasant spatial effects (e.g. spatial effects of phasiness)

User Interface

Each page contains 8 test recordings to be evaluated for spatial quality against a reference recording.

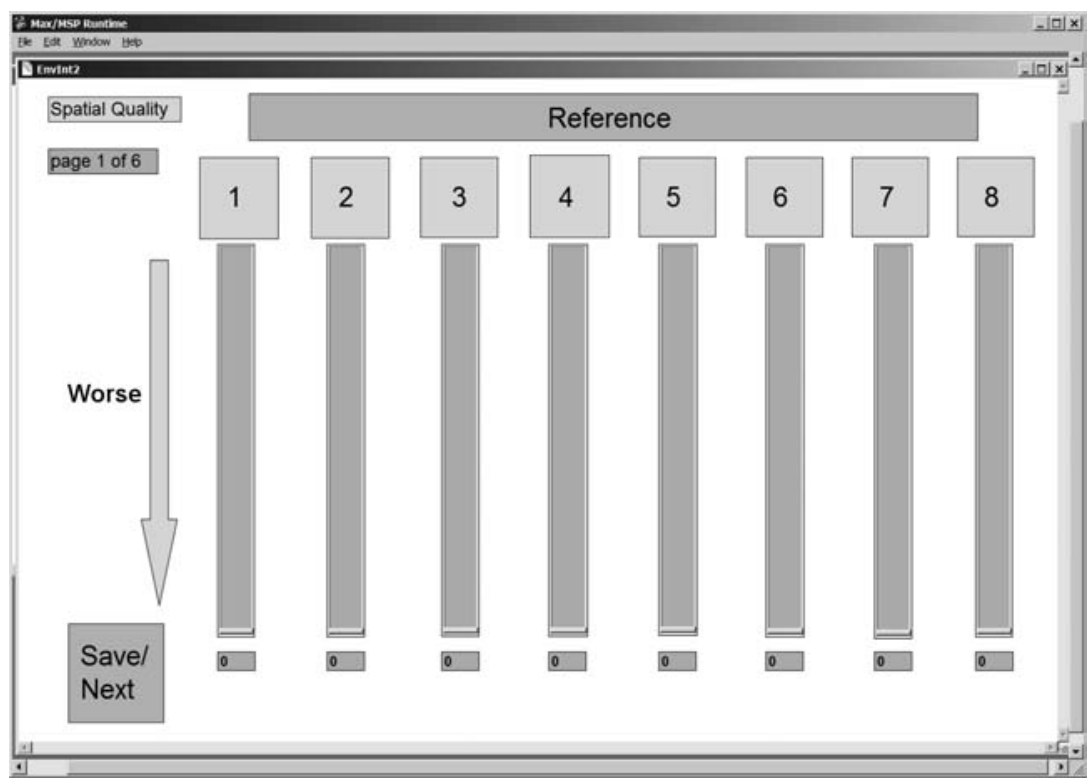

This experiment consists of 12 pages split over two parts, ' $a$ ' and 'b'.

When you come to the end of each part you will be prompted to save your responses. Please enter your initials followed by the test id (eg. RCa and $\mathrm{RCb}$ ).

Once you are happy with your responses click the save/next button to continue to the next page (NB. You'll we need to move each fader at least once (even if intend to return it to zero) before you can proceed to the next page).

Familiarisation

Before commencing the experiment you are required to complete a familiarisation session. This aims to familiarise you with the entire stimuli set that you will encounter in this study. Please think about how you would scale (rate) the spatial quality for each. 
Table 11. SAPs assessed at LP1 and LP 2 in listening test 1 and listening test 2 . All test items use 5 reproduction channels except where the description states otherwise (e.g., downmixing, channel removal)

\begin{tabular}{|c|c|c|c|c|c|c|}
\hline \multirow[b]{2}{*}{ SAP group } & \multirow[b]{2}{*}{ No. } & \multirow[b]{2}{*}{ Description } & \multicolumn{2}{|c|}{$\begin{array}{l}\text { Listening } \\
\text { test } 1\end{array}$} & \multicolumn{2}{|c|}{$\begin{array}{l}\text { Listening } \\
\text { test } 2\end{array}$} \\
\hline & & & LP1 & LP2 & LP1 & LP2 \\
\hline \multirow[t]{4}{*}{1} & 1 & 3/1 downmix: $\mathrm{L}=\mathrm{L}, \mathrm{R}=\mathrm{R}, \mathrm{C}=\mathrm{C}, \mathrm{S}=0.7071 * \mathrm{Ls}+0.7071 * \mathrm{Rs}$ & $\sqrt{ }$ & $\sqrt{ }$ & $\sqrt{ }$ & $\sqrt{ }$ \\
\hline & 2 & 3.0 downmix: $\mathrm{L}=\mathrm{L}+0.7071 * \mathrm{Ls}, \mathrm{R}=\mathrm{R}+0.7071 * \mathrm{Rs}, \mathrm{C}=\mathrm{C}$ & $\sqrt{ }$ & $\sqrt{ }$ & $x$ & $x$ \\
\hline & 3 & 2.0 downmix: $\mathrm{L}=\mathrm{L}+0.7071 * \mathrm{C}+0.7071 * \mathrm{Ls}, \mathrm{R}=\mathrm{R}+0.7071 * \mathrm{C}+0.7071 * \mathrm{Rs}$ & $\sqrt{ }$ & $\sqrt{ }$ & $\sqrt{ }$ & $\sqrt{ }$ \\
\hline & 4 & 1.0 downmix: $\mathrm{C}=0.7071 * \mathrm{~L}+0.7071 * \mathrm{R}+\mathrm{C}+0.5 * \mathrm{Ls}+0.5 * \mathrm{Rs}$ & $\sqrt{ }$ & $\sqrt{ }$ & $\sqrt{ }$ & $\sqrt{ }$ \\
\hline \multirow[t]{5}{*}{2} & 5 & Audio codec @ 160 kbs & $\sqrt{ }$ & $\sqrt{ }$ & $\sqrt{ }$ & $\sqrt{ }$ \\
\hline & 6 & Audio codec @ 64 kbs & $\sqrt{ }$ & $\sqrt{ }$ & $\sqrt{ }$ & $\sqrt{ }$ \\
\hline & 7 & Audio codec @ 64 kbs & $\sqrt{ }$ & $\sqrt{ }$ & $\sqrt{ }$ & $\sqrt{ }$ \\
\hline & 8 & 2 stage cascade $(80 \mathrm{kbs})$ & $\sqrt{ }$ & $\sqrt{ }$ & $x$ & $x$ \\
\hline & 9 & 4 stage cascade $(64 \mathrm{kbs})$ & $\sqrt{ }$ & $\sqrt{ }$ & $x$ & $x$ \\
\hline \multirow[t]{6}{*}{3} & 10 & $\mathrm{~L}$ and $\mathrm{R}$ re-positioned at $-10^{\circ}$ and $10^{\circ}$ & $\sqrt{ }$ & $\sqrt{ }$ & $x$ & $x$ \\
\hline & 11 & $\mathrm{C}$ is skewed; re-positioned at $20^{\circ}$ & $\sqrt{ }$ & $\sqrt{ }$ & $x$ & $x$ \\
\hline & 12 & Ls and Rs re-positioned at $-90^{\circ}$ and $90^{\circ}$ & $\sqrt{ }$ & $\sqrt{ }$ & $x$ & $x$ \\
\hline & 13 & Ls and Rs re-positioned at $-170^{\circ}$ and $160^{\circ}$ & $\sqrt{ }$ & $\sqrt{ }$ & $x$ & $x$ \\
\hline & 14 & $\mathrm{~L}$ and $\mathrm{C}$ moved $1 \mathrm{~m}$ to right and not facing listening position & $x$ & $x$ & $\sqrt{ }$ & $x$ \\
\hline & 15 & Ls moved $1 \mathrm{~m}$ to right and not facing listening position & $x$ & $x$ & $\sqrt{ }$ & $x$ \\
\hline \multirow[t]{4}{*}{4} & 16 & $\mathrm{~L}$ and $\mathrm{R}$ swapped & $\sqrt{ }$ & $\sqrt{ }$ & $\sqrt{ }$ & $\sqrt{ }$ \\
\hline & 17 & $\mathrm{~L}$ and $\mathrm{R}$ swapped for Ls and Rs & $\sqrt{ }$ & $\sqrt{ }$ & $x$ & $\dot{x}$ \\
\hline & 18 & Channel order rotated & $\sqrt{ }$ & $\sqrt{ }$ & $x$ & $x$ \\
\hline & 19 & Channel order randomised & $\sqrt{ }$ & $\sqrt{ }$ & $x$ & $x$ \\
\hline \multirow[t]{2}{*}{5} & 20 & $\mathrm{~L}, \mathrm{C}$ and $\mathrm{R}$ each attenuated by $6 \mathrm{~dB}$ & $\sqrt{ }$ & $\sqrt{ }$ & $\sqrt{ }$ & $\sqrt{ }$ \\
\hline & 21 & Ls and Rs each attenuated by $6 \mathrm{~dB}$ & $x$ & $x$ & $\sqrt{ }$ & $\sqrt{ }$ \\
\hline \multirow[t]{2}{*}{6} & 22 & C phase-inverted & $\sqrt{ }$ & $\sqrt{ }$ & $\sqrt{ }$ & $\sqrt{ }$ \\
\hline & 23 & $\mathrm{~L}, \mathrm{C}$ and $\mathrm{R}$ phase-inverted & $x$ & $x$ & $\sqrt{ }$ & $\sqrt{ }$ \\
\hline \multirow[t]{3}{*}{7} & 24 & $\mathrm{R}$ removed & $\sqrt{ }$ & $\sqrt{ }$ & $x$ & $x$ \\
\hline & 25 & Ls removed & $\sqrt{ }$ & $\sqrt{ }$ & $x$ & $x$ \\
\hline & 26 & C removed & $\sqrt{ }$ & $\sqrt{ }$ & $\sqrt{ }$ & $\sqrt{ }$ \\
\hline \multirow[t]{2}{*}{8} & 27 & $500 \mathrm{~Hz} \mathrm{HPF}$ on all channels & $\sqrt{ }$ & $\sqrt{ }$ & $\sqrt{ }$ & $\sqrt{ }$ \\
\hline & 28 & $3.5 \mathrm{kHz}$ LPF on all channels & $\sqrt{ }$ & $\sqrt{ }$ & $\sqrt{ }$ & $\sqrt{ }$ \\
\hline \multirow[t]{2}{*}{9} & 29 & 1.0 downmix in all channels & $\sqrt{ }$ & $\sqrt{ }$ & $\sqrt{ }$ & $\sqrt{ }$ \\
\hline & 30 & Partly correlated ( 0.5 bleed in adjacent channel pairs) & $\sqrt{ }$ & $\sqrt{ }$ & $x$ & $x$ \\
\hline \multirow[t]{2}{*}{10} & 31 & Line array virtual surround & $\sqrt{ }$ & $\sqrt{ }$ & $x$ & $x$ \\
\hline & 32 & 2 channel virtual surround & $\sqrt{ }$ & $\sqrt{ }$ & $x$ & $x$ \\
\hline \multirow[t]{13}{*}{11} & 33 & Channel order randomised $+\mathrm{R}$, Ls and $\mathrm{C}$ removed & $\sqrt{ }$ & $\sqrt{ }$ & $x$ & $x$ \\
\hline & 34 & 3.0 downmix $+\mathrm{R}$ removed & $\sqrt{ }$ & $\sqrt{ }$ & $x$ & $x$ \\
\hline & 35 & 2.0 downmix + channel order randomised & $\sqrt{ }$ & $\sqrt{ }$ & $x$ & $x$ \\
\hline & 36 & 2.0 downmix $+\mathrm{L}$ and $\mathrm{R}$ re-positioned at $-10^{\circ}$ and $10^{\circ}$ & $\sqrt{ }$ & $\sqrt{ }$ & $x$ & $x$ \\
\hline & 37 & 1.0 downmix $+500 \mathrm{~Hz} \mathrm{HPF}$ on all channels & $\sqrt{ }$ & $\sqrt{ }$ & $\sqrt{ }$ & $\sqrt{ }$ \\
\hline & 38 & $\mathrm{~L}$ and $\mathrm{R}$ re-positioned at $-10^{\circ}$ and $10^{\circ}+\mathrm{Ls}$ and Rs re-positioned at $-170^{\circ}$ and $160^{\circ}$ & $\sqrt{ }$ & $\sqrt{ }$ & $x$ & $x$ \\
\hline & 39 & Audio codec $-160 \mathrm{kbs}+2.0$ downmix & $\sqrt{ }$ & $\sqrt{ }$ & $\sqrt{ }$ & $\sqrt{ }$ \\
\hline & 40 & Audio codec $-160 \mathrm{kbs}+\mathrm{Ls}$ and Rs re-positioned at $-90^{\circ}$ and $90^{\circ}$ & $\sqrt{ }$ & $\sqrt{ }$ & $x$ & $x$ \\
\hline & 41 & Audio codec @ 64 kbs + 1.0 downmix & $\sqrt{ }$ & $\sqrt{ }$ & $x$ & $x$ \\
\hline & 42 & Audio codec@64 kbs + channel order randomised & $\sqrt{ }$ & $\sqrt{ }$ & $x$ & $x$ \\
\hline & 43 & 2 channel virtual surround $+R$ removed & $\sqrt{ }$ & $\sqrt{ }$ & $x$ & $x$ \\
\hline & 44 & 2 channel virtual surround $+\mathrm{L}$ and $\mathrm{R}$ re-positioned at $-10^{\circ}$ and $10^{\circ}$ & $\sqrt{ }$ & $\sqrt{ }$ & $x$ & $x$ \\
\hline & 45 & Audio codec @ $64 \mathrm{kbs}+$ Ls moved $1 \mathrm{~m}$ to right and not facing listening position & $x$ & $x$ & $\sqrt{ }$ & $\sqrt{ }$ \\
\hline \multirow[t]{3}{*}{12} & 46 & High Anchor: unprocessed reference & $\sqrt{ }$ & $\sqrt{ }$ & $\sqrt{ }$ & $\sqrt{ }$ \\
\hline & 47 & Mid Anchor: audio codec $(80 \mathrm{kbs})$ & $\sqrt{ }$ & $\sqrt{ }$ & $\sqrt{ }$ & $\sqrt{ }$ \\
\hline & 48 & Low Anchor: mono downmix reproduced asymmetrically by Ls only & $\sqrt{ }$ & $\sqrt{ }$ & $\sqrt{ }$ & $\sqrt{ }$ \\
\hline
\end{tabular}




\section{THE AUTHORS}

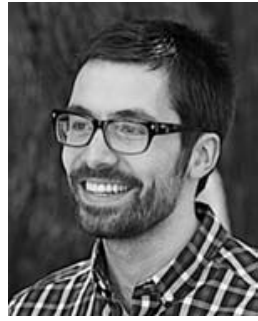

Robert Conetta

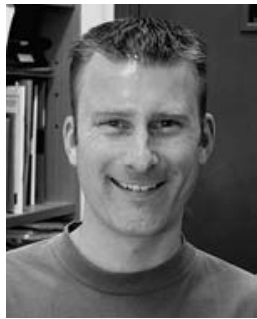

Tim Brookes

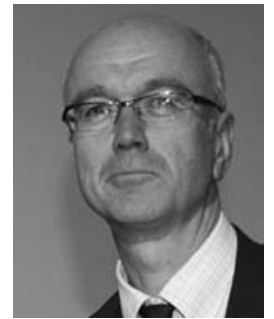

Francis Rumsey

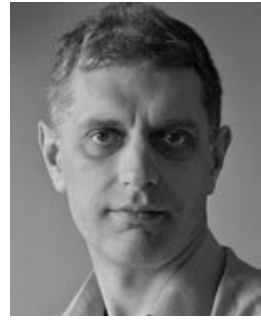

Sławomir Zieliński

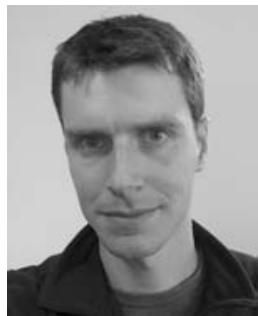

Martin Dewhirst

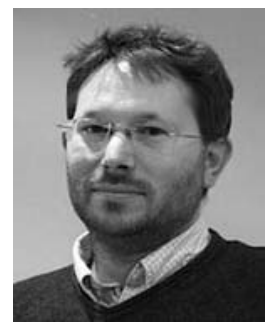

Philip Jackson

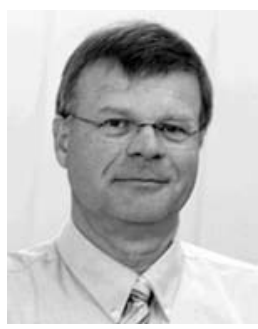

Søren Bech

Robert Conetta is an acoustics engineer at Sandy Brown Associates LLP. Previously he was an acoustics consultant at Marshall Day Acoustics and a research fellow at the Acoustics Research Centre, London South Bank University. At LSBU he worked with Professor Bridget Shield, Professor Julie Dockrell (IOE), and Professor Trevor Cox (Salford) to investigate the effect of noise and classroom acoustic design on pupil performance on the ISESS project.

Rob studied for his Ph.D. at the Institute of Sound Recording, University of Surrey under the supervision of Professor Francis Rumsey, Dr. Sławomir Zieliński, and Dr. Tim Brookes. He worked as part of a team of researchers, funded and supported by Bang and Olufsen and BBC research, on the QESTRAL (Quality Evaluation of Spatial Transmission and Reproduction using an Artificial Listener) project. For his contribution to the project he received University of Surrey's Research Student of the Year Award in 2010.

Tim Brookes received the B.Sc. degree in mathematics and the M.Sc. and D.Phil. degrees in music technology from the University of York, York, U.K., in 1990, 1992, and 1997, respectively. He was employed as a software engineer, recording engineer, and research associate before joining, in 1997, the academic staff at the Institute of Sound Recording, University of Surrey, Guildford, U.K., where he is now senior lecturer in audio and director of research. His teaching focuses on acoustics and psychoacoustics and his research is in psychoacoustic engineering: measuring, modeling, and exploiting the relationships between the physical characteristics of sound and its perception by human listeners.

Francis Rumsey is an independent technical writer and consultant, based in the U.K. Until 2009 he was professor

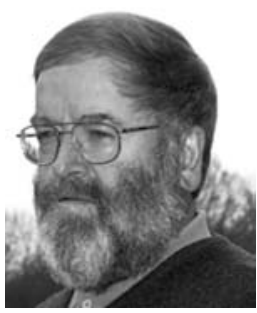

David Meares

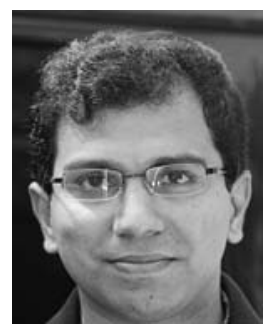

Sunish George and director of research at the Institute of Sound Recording, University of Surrey, specializing in sound quality, psychoacoustics, and spatial audio. He led the QESTRAL project on spatial sound quality evaluation from 2006-9. $\mathrm{He}$ is currently chair of the AES Technical Council, Consultant Technical Writer, and Editor for the AES Journal. Among his musical activities he is organist and choirmaster of St. Mary the Virgin Church in Witney, Oxfordshire.

Sławomir Zieliński received M.Sc. and Ph.D. degrees in telecommunications from the Technical University of Gdańsk, Poland. After graduation in 1992, he worked as a lecturer at the same University for eight years. In 2000 Dr. Zieliński joined the University of Surrey, U.K., where he initially worked as a research fellow and then as a lecturer at the Department of Music and Sound Recording. Since 2009 he has been working as a teacher at the Technical Schools in Suwałki, Poland.

During the past 20 years Dr. Zieliński taught classes in a broad range of topics including electronics, electroacoustics, audio signal processing, sound synthesis, studio recording technology, and more recently information and communications technology. He co-supervised six Ph.D. students. In 2007-2008 he was a member of the AES British Section Committee. He is the author or co-author of more than 70 scientific papers in the area of audio engineering. His current research interests include psychoacoustics and audio quality assessment methodology.

Martin Dewhirst received an MMath degree from the University of Manchester Institute of Science and Technology, Manchester, U.K., and a Ph.D. degree from the Institute of Sound Recording and the Centre for Vision, Speech and Signal Processing at the University of Surrey, Guildford, UK. 
$\mathrm{He}$ is a lecturer at the Institute of Sound Recording, University of Surrey, where his teaching focuses on signal processing and sound synthesis. His current research interests include the relationship between audio quality and lower level perceptual attributes and modeling the perceived attributes of reproduced sound using objective measurements. Dr. Dewhirst is an associate member of the Audio Engineering Society.

Philip Jackson is senior lecturer in speech and audio processing at the Centre for Vision, Speech \& Signal Processing (University of Surrey, U.K.) which he joined in 2002, following a postdoctoral research fellowship (University of Birmingham, U.K.), with MA in Engineering (Cambridge University, U.K.) and Ph.D. in electronic engineering (University of Southampton, U.K.). With Dr. Wenwu Wang in CVSSP, he leads the Machine Audition Group (A-lab) of around a dozen research fellows and students. His research in acoustical and spoken-language processing has contributed to various projects (e.g., BALTHASAR, DANSA, Dynamic Faces, QESTRAL, UDRC, POSZ, and S3A) in active noise control for aircraft, acoustics of speech production, source separation for automatic speech recognition (ASR), use of articulatory models for ASR, audio-visual processing for speech enhancement and visual speech synthesis, as well as spatial aspects of subjective sound quality evaluation. He has over 100 academic publications in journals, conference proceedings, and books. He reviews for journals and conferences including Journal of the Acoustical Society of America, IEEE Transactions on Audio, Speech \& Language Processing, IEEE Signal Processing Letters, InterSpeech and ICASSP, and is associate editor for Computer Speech \& Language (Elsevier).

Søren Bech received a M.Sc. and a Ph.D. from the Department of Acoustic Technology (AT) of the Technical University of Denmark. From 1982-92 he was a research Fellow at AT studying perception and evaluation of reproduced sound in small rooms. In 1992 he joined Bang
\& Olufsen where he is head of research. In 2011 he was appointed professor in audio perception at Aalborg University.

His research interest includes human perception of reproduced sound in small and medium sized rooms, experimental procedures, and statistical analysis of data from sensory analysis of audio and video quality. General perception of sound in small rooms is also a major research interest.

David Meares is a graduate in electrical engineering from Salford University, Salford, U.K. In his 38 years at the $\mathrm{BBC}$, he rose to be head of the studio group. He led a wide range of projects including acoustic scale modeling, digital television, applications of speech recognition, display technology, surround sound, and compression coding. He represented the $\mathrm{BBC}$ in a number of international standards groups and on international collaborative projects. This broad experience suits him ideally for the wide number of tasks he has been doing for International Broadcasting Convention over many years. Since introducing the idea 16 years ago, he has organized the New Technology Campus and has served on the papers committee and at various times on the management committee and the conference committee.

Sunish George received the B.Tech degree from Cochin University of Science and Technology, Kerala, in 1999, and the M.Tech degree in digital electronics and advanced communication from Manipal Institute of Technology, Karnataka, in 2003. After his graduations he worked in various Indian software companies developing digital signal processing-based applications. He completed his Ph.D. from the Institute of Sound Recording, University of Surrey in July 2009. The focus of his doctoral work was to contribute towards the development of a generic objective model that predicts multichannel audio quality. He is currently working at Harman Becker Automotive Systems GmbH, Germany. 